Document downloaded from:

http://hdl.handle.net/10251/70971

This paper must be cited as:

ALI; Juan-Carlos Cano; Tavares De Araujo Cesariny Calafate, CM.; Manzoni ., P. (2013). Reducing Channel Contention in Vehicular Environments Through an Adaptive Contention Window Solution. IEEE IEEE Catalog Number CFP1314F-USB. 1-6.

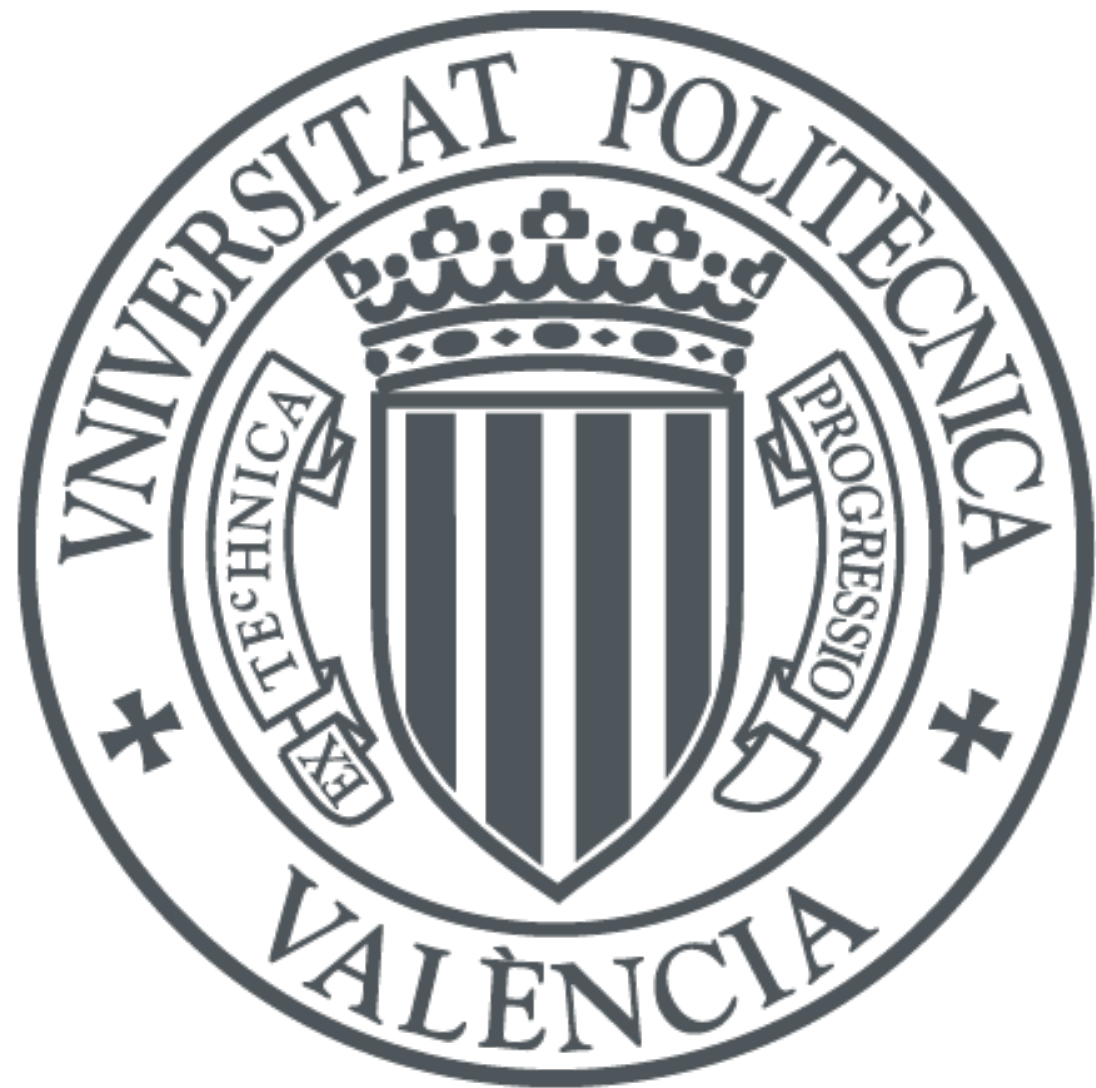

The final publication is available at

http://dx.doi.org/10.1109/WD.2013.6686512

Copyright IEEE

Additional Information

(C) 2013 IEEE. Personal use of this material is permitted. Permission from IEEE must be obtained for all other uses, in any current or future media, including reprinting/republishing this material for advertising or promotional purposes, creating new collective works, for resale or redistribution to servers or lists, or reuse of any copyrighted component of this work in other works. 


\title{
Reducing Channel Contention in Vehicular Environments Through an Adaptive Contention Window Solution
}

\author{
Ali Balador, Carlos T. Calafate, Juan-Carlos Cano and Pietro Manzoni \\ Universitat Politecnica de Valencia \\ Camino de Vera, s/n, 46022 Valencia, Spain \\ Email: alba6@upv.es, calafate, jucano,pmanzoni@disca.upv.es
}

\begin{abstract}
Intelligent Transportation Systems (ITS) are attracting growing attention both in industry and academia due to the advances in wireless communication technologies, and a significant demand for a wide variety of applications targeting this kind of environments are expected. In order to make it usable in real vehicular environments, achieving a well-designed Medium Access Control (MAC) protocol is a challenging issue due to the dynamic nature of Vehicular Ad Hoc Networks (VANETs), scalability issues, and the variety of application requirements. Different standardization organizations have selected IEEE 802.11 as the first choice for VANET environments considering its availability, maturity, and cost. The contention window is a critical parameter for handling medium access collisions by the IEEE 802.11 MAC protocol, and it highly affects the communications performance. The impact of adjusting the contention window has been studied in Mobile Ad-Hoc Networks (MANETs), but the vehicular communications community has not yet addressed this issue thoroughly. This paper proposes a new contention window control scheme, called DBM-ACW, for VANET environments. Analysis and simulation results using OMNeT++ in a highway scenario show that DBM-ACW provides better overall performance compared with previous proposals, even with high network densities.
\end{abstract}

\section{INTRODUCTION}

Advances in wireless communication technologies, along with the increasing demand for a wide variety of applications, make Vehicular Ad Hoc Networks (VANETs) an attractive research area in both academia and industry. VANETs are formed by vehicles equipped with wireless devices in order to communicate with other vehicles in infrastructure-less wireless networks (vehicle to vehicle, V2V) or with roadside units (vehicle to infrastructure, V2I). Vehicular environments with inter-vehicle communications can be assumed as a special form of Mobile Ad-Hoc Networks (MANETs) in which nodes are road-constrained [1].

The main differences between VANETs and MANETs have to do with rapid topology changes and diversity of network scenarios. For example, in highway scenarios, vehicles have mostly one straightforward direction, but high relative speeds up to $100 \mathrm{~km} / \mathrm{h}$ that can lead to frequent network disconnections. On the other hand, in urban scenarios, highdensity networks appear during rush hours. Also, network disconnection can occur in the late night hours or idle daytime hours. Due to these issues, designing protocols that can satisfy different types of scenarios is still an open issue.

Medium Access Control protocols play an important role since critical communications must rely on it. Unfortunately, research results [2] highlight that the topic of MAC support in VANETs has received less attention than other research fields. Also, most of these relatively few research works are dedicated to V2I communications; therefore, MAC support for $\mathrm{V} 2 \mathrm{~V}$ communication needs more attention. MAC layer design challenges in VANET environments can be summarized as follows [3]: (a) achieving an effective channel access coordination in the presence of changing vehicle locations and variable channel characteristics; (b) supporting scalability in the presence of various traffic densities; and (c) supporting a diverse set of application requirements.

A lot of research has been done by the research community with the idea of supporting broadcast transmissions in mind (e.g, [4]-[6]). In contrast to the most common research trend, this paper targets unicast applications including infotainment, $\mathrm{P} 2 \mathrm{P}$ or VoIP.

The IEEE 802.11 has been selected by a wide range of research works for vehicular environments as the MAC layer standard because of its availability, maturity, and cost. However, it causes performance to be poor in VANETs compared to MANET environments as a result of the differences between these two networks. A well-known problem in IEEE 802.11 is scalability, which becomes more challenging in VANETs in the presence of high and variable network densities. A lot of works have been proposed and carried out for MANET environments [7]-[11] to either solve or reduce this problem.

Among these studies, a dynamic and low-overhead method called HBCWC is proposed in [11]. This method estimates network density based on channel status observations without requiring complex calculations. This way, more than current network status, previous statuses are used in order to identify the channel traffic variations. As a consequence, the CW size is dynamically tuned based on the estimated network density.

However, to the best of our knowledge, very few studies address this topic in vehicular environments. A fuzzy logic based enhancement to $802.11 \mathrm{p}$ is proposed in [12] which adapts the $\mathrm{CW}$ size based on a non-linear control law, and relies on channel observation. Furthermore, [13] suggests a MAC mechanism which uses a modified version of RTS/CTS in order to estimate network density through message exchange. Therefore, in this paper we propose a new contention window control scheme, called DBM-ACW (Density Based Method for Adjusting the CW size), and prove that DBM-ACW not only outperforms the IEEE 802.11 DCF in different vehicular scenarios, but also previously proposed schemes.

The rest of this paper is organized as follows. In section 


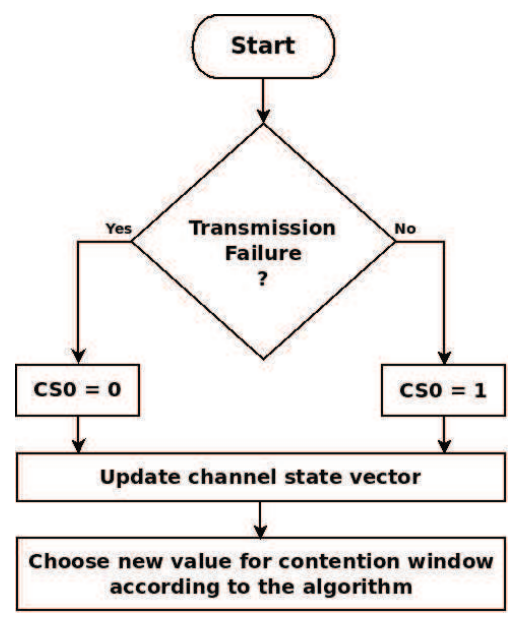

Fig. 1. CS vector updating in DBM-ACW.

II, we describe the new proposed contention window control scheme in detail. Performance evaluation of DBM-ACW, including simulation results in a highway scenario, is presented in section III. Finally, section IV concludes this paper.

\section{The Proposed Algorithm}

In order to adequately adjust the $\mathrm{CW}$ size for vehicular environments, we propose DBM-ACW, a new method to select the CW size based on the network traffic density. In this method, the channel condition is estimated based on the packet transmission status, and the result is stored into a Channel State (CS) vector. A significant part of the protocol relies on how the channel conditions are captured by the CS vector, and how this vector is used to update the $\mathrm{CW}$ size in order to improve throughput, which it is the key contribution of this paper. These two issues will be further explained in the following sections.

\section{A. The Channel State Vector}

In legacy 802.11 DCF, and after each data frame transmission, each node sets its timer and waits for an acknowledgement. In DBM-ACW, if the transmitter receives an ACK frame from the receiver, a value of 1 is inserted into the channel state vector. Otherwise, if a collided/faulty frame is received, or if the transmitter waiting timer expires before receiving the acknowledgement, a value of 0 is inserted into the channel vector.

Upon each channel state change notification, the CS vector is updated by shifting to the left. Based on extensive simulations, we chose a three-element array for DBM-ACW in order to achieve a trade-off between overhead and performance. If we choose a smaller array, it will not be able to reflect the real network condition, while larger array values do not lead to a significant performance improvement. Figure 1 shows the CS vector updating strategy in DBM-ACW.

\section{B. Changing the Contention Window Size}

In the proposed scheme, upon each frame loss, timer expiration or collision, the $\mathrm{CW}$ size is doubled (similarly to IEEE 802.11 DCF) in order to obtain the highest PDR, except for the case in which the CS array contains two consecutive ones before the new state; in that case the $\mathrm{CW}$ is multiplied by $\mathrm{A}$. Furthermore, the $\mathrm{CW}$ size is set to the minimum $\mathrm{CW}$, $C W_{\min }$, upon each acknowledgement reception, except for the case in which the CS array contains two consecutive zeros before the new state; in that case the $\mathrm{CW}$ is multiplied by parameter B.

Table I details how the CW size is chosen for each CS array state in DBM-ACW. The table was achieved based on the extensive simulations in which different combinations of values were used to obtain the best performance. According to the severity of channel congestion, the current $\mathrm{CW}$ size is multiplied by a value in the range from 0.2 to 2 or set to $C W_{\min }$. The upper bound is selected as in IEEE 802.11 DCF, so that the CW size is multiplied by 2 when the channel is detected as busy or a collision has occurred. When the channel is very congested, the current $\mathrm{CW}$ size is multiplied by a value in the upper part of this range in order to decrease the probability of selecting the same backoff number. Otherwise, when the channel density is low, the current $\mathrm{CW}$ size is multiplied by a value in the lower range or set to $C W_{\min }$ in order to avoid waiting for a long time when the channel occupation is low. In our study, the optimal value for parameters $\mathrm{A}$ and $\mathrm{B}$ was found to be equal to 1.7 and 0.8 , respectively.

TABLE I. CONTENTION WINDOW SIZE UPDATING

\begin{tabular}{ll}
\hline Status & CW range \\
\hline \hline 000 & \\
010 & $C W_{\text {old }} * 2$ \\
100 & \\
\hline 110 & $C W_{\text {old }} * \mathrm{~A}$ \\
\hline 001 & $C W_{\text {old }} * \mathrm{~B}$ \\
\hline 011 & \\
101 & $C W_{\text {min }}$ \\
111 & \\
\hline
\end{tabular}

\section{Simulation}

In this section, we study the performance of DBM-ACW in comparison with IEEE 802.11 DCF and HBCWC in vehicular environments by using the $\mathrm{OMNeT}++$ discrete event simulator (version 4.2.2) [14]. We use this simulator coupled with the INETMANET framework [15] and SUMO [16] in order to provide a realistic vehicular scenario. The INETMANET framework provides detailed models for simulating wireless networks in OMNeT++ such as wireless channels, connectivity, mobility and especially MAC layer protocols. Also, SUMO is used to generate real vehicular traffic in road networks.

\section{A. Simulation Parameters}

Each vehicle generates constant bitrate traffic. The size of the data payload is 512 bytes, and each node generates data packets at a rate of 2 packets per second. Each vehicle starts a new connection after joining the network, and sends its packets to a randomly selected destination among the current vehicles in the network. Also, each vehicle immediately changes its destination when the destination leaves the network.

Considering the routing protocol, we assessed different routing protocols and, despite of the different overall performance levels obtained by these protocols, we found that they experience the same impact when combined with the different MAC protocols which are evaluated in this paper. As a consequence, we chose the AODV routing protocol, which is a simple routing protocol that can be easily implemented when attempting to test it in practical scenarios. The radio propagation range for each node is set to $250 \mathrm{~m}$. We used 
TABLE II. THE SIMULATION PARAMETERS

\begin{tabular}{ll}
\hline Simulation Parameter & Value \\
\hline \hline Traffic type & CBR \\
CBR packet size & 512 byte \\
CBR data rate & 2 packet/s \\
Transport protocol & UDP \\
Routing protocol & AODV \\
\hline MAC protocol & $802.11 \mathrm{a}$ \\
Max. and Min. of CW & 7,1023 \\
Max. number of retransmissions & 7 \\
RTS/CTS threshold & 2346 byte \\
\hline Max. transmission range & $250 \mathrm{~m}$ \\
Propagation model & Nakagami \\
Nakagami-m & 0.7 \\
\hline Simulation time & 300 seconds \\
Number of repetitions & 10 \\
\hline
\end{tabular}

the Nakagami radio propagation model, commonly used by the VANET community, in order to present a more realistic vehicular environment [17]. Parameter $m$ for this propagation model is set to 0.7 . Moreover, each point in the figures that follow represents the average of 10 independent simulation experiments in which the simulation time is 300 seconds. Table II summarizes the simulation parameters.

The highway scenario models a $4 \mathrm{~km}$ highway with 3 lanes, where the lane width is $3 \mathrm{~m}$. The destination is randomly chosen, so it can be a car ahead or behind the transmitter. In order to model a realistic scenario, we assumed that vehicles can be selected based on a normal distribution from three different categories: fast, normal, and slow. For these categories, the maximum speeds $(\mathrm{m} / \mathrm{s})$ are set to $36,28,20$, and selection probabilities are set to $10 \%, 80 \%$, and $10 \%$, respectively. Moreover, vehicles are injected in the highway according to a Poisson process with a mean interval time of 2 seconds. In each graph the probability of sending a message defines the transmission probability for each vehicle throughout the simulation.

The metrics used to evaluate the performance of the proposed scheme are the following: (a) Packet Delivery Ratio (PDR), which represents the ratio between the total number of packets received by the final destination and the number of packets originated by the source; (b) average end-to-end delay, which represents the average time required for a packet to travel from source to destination; and (c) average MAC collisions, which shows the average number of collisions experienced per source.

\section{B. Result and Analysis}

Concerning the experimental results obtained, Figure 2 shows a clear packet delivery ratio improvement for DBMACW in comparison to IEEE 802.11, achieved by adapting the $\mathrm{CW}$ size based on the channel history. As a consequence, it reduces $\mathrm{CW}$ size variations since increasing the $\mathrm{CW}$ size starting from $C W_{\min }$ is no longer required to find the optimal CW size. For example, IEEE 802.11 sets the CW size value to the $C W_{\min }$ after reaching the retransmission limit due to consecutive transmission failures, which further aggravates channel contention problems. DBM-ACW avoids changing the $\mathrm{CW}$ size and starts the new transmission with the current $\mathrm{CW}$ size, so it shows a better performance compared with IEEE 802.11 .

Furthermore, DBM-ACW outperforms HBCWC by not resetting the $\mathrm{CW}$ size to $C W_{\min }$ when the $\mathrm{CS}$ array contains two consecutive zeros before a successful transmission. The average number of MAC collisions, shown in Figure 3, offers a hint on how to achieve improvements in terms of PDR. As can be observed in this figure, the optimal CW size for DBM-ACW was chosen so that it decreases the probability of picking the same backoff value, and consequently the number of collisions is also reduced. Also, Figure 2 shows that, in denser networks, and when the PDR severely goes down for HBCWC and IEEE 802.11, DBM-ACW shows a much smoother decrease.



Fig. 2. PDR for the highway scenario.

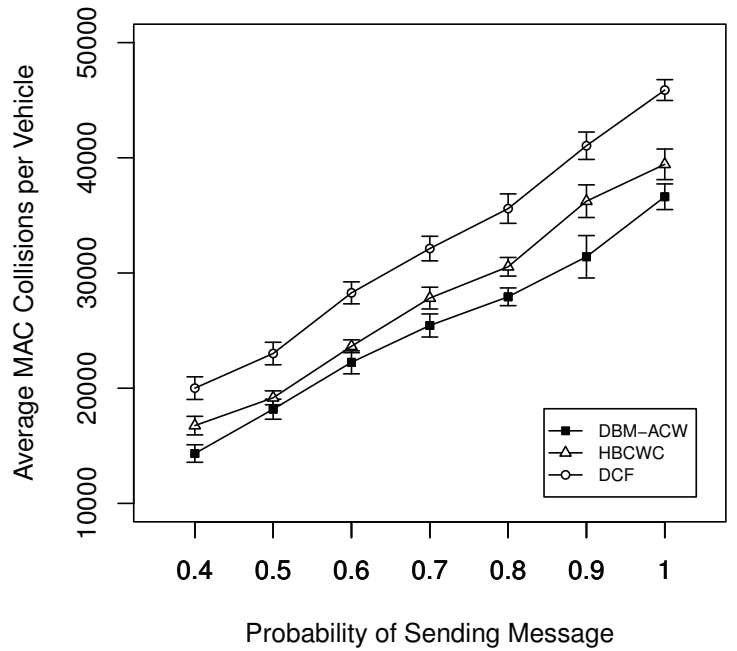

Fig. 3. Average number of collisions for the highway scenario.

Figure 4 shows that HBCWC achieves slightly better delay when compared with DBM-ACW. However, DBM-ACW achieves a better trade-off between PDR and end-to-end delay, meaning that the total PDR improvement ratio is higher than the delay degradation ratio when compared with HBCWC.

Overall, we obtain a $20 \%$ improvement in terms of PDR 




Fig. 4. Average end-to-end delay for the highway scenario.

and a $22 \%$ improvement in terms of end-to-end delay compared with IEEE $802.11 \mathrm{DCF}$, as well as a $7 \%$ improvement in terms of PDR compared with HBCWC.

\section{CONCLUSION}

The impact of controlling the contention window on the performance of the IEEE 802.11 MAC is an issue that has been studied by the MANET research community, remaining mostly untackled in VANET environments. In this paper we propose a new IEEE 802.11-based MAC protocol which controls the CW size based on a network density estimation. Each node in DBM-ACW stores its transmission trials history in the network as an array which is used to determine the optimal contention window value. We focused on highway environments to demonstrate the performance benefits of DBM-ACW. Simulation results in these scenarios prove that our scheme has better overall performance compared with IEEE 802.11 DCF and $\mathrm{HBCWC}$ in terms of PDR, end-to-end delay and average number of collisions.

As a future work, we will study the effect of the existence of RSUs on the performance of our scheme. Also, the algorithm's parameters can be dynamically controlled based on the specific network scenario.

\section{ACKNOWLEDGMENT}

This work was partially supported by the Ministerio de Ciencia e Innovación, Spain, under Grant TIN2011-27543C03-01.

\section{REFERENCES}

[1] Hartenstein, H.; Laberteaux, K.P., "A tutorial survey on vehicular ad hoc networks," Communications Magazine, IEEE , vol.46, no.6, pp.164,171, June 2008.

[2] Booysen, M.J.; Zeadally, S.; van Rooyen, G.-J., "Survey of media access control protocols for vehicular ad hoc networks," Communications, IET , vol.5, no.11, pp.1619,1631, July 222011.

[3] Kenney, J.; "Standards and regulations," in Hartenstein, H., Laberteaux, K.P. (Eds.): "VANET: vehicular applications and inter-networking technologies" (Wiley, 2010), Ch. 10, pp. 365428.

[4] Omar, H.; Zhuang, W.; Li, L., "VeMAC: A TDMA-based MAC Protocol for Reliable Broadcast in VANETs," Mobile Computing, IEEE Transactions on , vol.PP, no.99, pp.1,1, 0 doi: 10.1109/TMC.2012.142.

[5] Stanica, R.; Chaput, E.; Beylot, A.-L., "Enhancements of IEEE 802.11p Protocol for Access Control on a VANET Control Channel," Communications (ICC), 2011 IEEE International Conference on , vol., no., pp.1,5, 5-9 June 2011.

[6] Djahel, S.; Ghamri-Doudane, Y., "A robust congestion control scheme for fast and reliable dissemination of safety messages in VANETs," Wireless Communications and Networking Conference (WCNC), 2012 IEEE , vol., no., pp.2264,2269, 1-4 April 2012.

[7] Cali, F.; Conti, M.; Gregori, E., 'Dynamic tuning of the IEEE 802.11 protocol to achieve a theoretical throughput limit," Networking, IEEE/ACM Transactions on , vol.8, no.6, pp.785,799, Dec 2000.

[8] Bianchi, G.; Tinnirello, I., "Kalman filter estimation of the number of competing terminals in an IEEE 802.11 network," INFOCOM 2003. Twenty-Second Annual Joint Conference of the IEEE Computer and Communications. IEEE Societies, vol.2, no., pp.844,852 vol.2, March 30 2003-April 32003.

[9] Wu, H.; Cheng, S.; Peng, Y.; Long, K.; Ma, J., "IEEE 802.11 distributed coordination function (DCF): analysis and enhancement," Communications, 2002. ICC 2002. IEEE International Conference on , vol.1, no., pp.605,609, 2002.

[10] Ni, Q; Aad, I.; Barakat, C.; Turletti, T., "Modeling and analysis of slow CW decrease IEEE 802.11 WLAN," Personal, Indoor and Mobile Radio Communications, 2003. PIMRC 2003. 14th IEEE Proceedings on , vol.2, no., pp.1717,1721 vol.2, 7-10 Sept. 2003.

[11] Balador, A.; Movaghar, A.; Jabbehdari, S., "History based contention window control in ieee 802.11 mac protocol in error prone channel," Journal of Computer Science, vol.6, no.2, pp.205,209, 2010.

[12] Chrysostomou, C.; Djouvas, C.; Lambrinos, L., "Applying adaptive QoS-aware medium access control in priority-based vehicular ad hoc networks," Computers and Communications (ISCC), 2011 IEEE Symposium on , vol., no., pp.741,747, June 28 2011-July 12011.

[13] Jang, H.-C.; Feng, W.-C., "Network Status Detection-Based Dynamic Adaptation of Contention Window in IEEE 802.11p," Vehicular Technology Conference (VTC 2010-Spring), 2010 IEEE 71st, vol., no., pp.1,5, 16-19 May 2010.

[14] Website: http://www.omnetpp.org/

[15] Website: http://inet.omnetpp.org/

[16] Behrisch, M.; Bieker, L.; Erdmann, J.; Krajzewicz, D., SUMO Simulation of Urban MObility: An Overview In: SIMUL 2011, The Third International Conference on Advances in System Simulation, 2011.

[17] Baguena, M.; Calafate, C.T.; Cano, J.; Manzoni, P., "Towards realistic vehicular network simulation models," Wireless Days (WD), 2012 IFIP , vol., no., pp.1,3, 21-23 Nov. 2012. 